Pacific Journal of Mathematics

ASYMPTOTIC ENUMERATION OF PARTIALLY ORDERED
SETS 


\section{ASYMPTOTIC ENUMERATION OF PARTIALLY ORDERED SETS}

\section{DEEPAK DHAR}

We define the entropy function $S(\rho)=\operatorname{Lim}_{n-\infty} 2 n^{-2} \ln N(n, \rho)$, where $N(n, o)$ is the number of distinct partial order relations which may be defined on a set of $n$ elements such that a fraction $\rho$ of the possible $n(n-1) / 2$ pairs are comparable. We derive upper bounds to $S(\rho)$ to show that $S(\rho)<(1 / 2) \ln 2$ if $\rho \geq$. 699 .

I. Introduction. In an earlier paper [1, hereafter referred to as I] we have studied the asymptotic enumeration of partial order relations defined over a set of $n$ distinct objects, subject to a constraint that a given fraction $\rho$ of the $n(n-1) / 2$ pairs are comparable. Let this number be denoted by $N(n, \rho)$. We showed that $N(n, \rho)$ increases as an exponential of $n^{2}$ for large $n$ (except in the trivial cases, when $\rho$ is either zero or one), and defined a function $S(\rho)$ by the equation

$$
S(\rho)=\operatorname{Lim}_{n \rightarrow \infty} 2 n^{-2} \ln N(n, \rho) .
$$

This function $S(\rho)$ may be called the entropy function as it is related to the thermodynamic entropy of a lattice-gas with a longrange three-body interaction. For details of this equivalence, the reader is referred to $I$.

Using upper and lower bounds on $S(\rho)$, we showed that $S(\rho)$ is a continuous function of $\rho$ for the allowed range of $\rho, 0 \leqq \rho \leqq 1$. It is, however, not an analytic function of $\rho$. It was proved that

$$
S(\rho)=\frac{1}{2} \ln 2 \quad \text { if } \quad \frac{1}{4} \leqq \rho \leqq \frac{3}{8}
$$

and

$$
S(\rho)<\frac{1}{2} \ln 2, \quad \text { if } \rho \leqq .083 \text { or if } \rho \geqq 48 / 49 \text {. }
$$

The equality in (2) could be proved, because in this range of $\rho$, we derived a lower bound to $S(\rho)$ which coincides with an earlier known $\rho$-independent upper bound due to Kleitmen and Rothschild [2]. We conjectured that the lower bound (derived in I) gives the exact value of $S(\rho)$ for all $\rho$. This, however, could not be proved because the corresponding upper bounds to $S(\rho)$ were too weak. We have subsequently improved the upper bounds. Using these improved bounds we can show that 


$$
S(\rho)<\frac{1}{2} \ln 2, \quad \text { if } \rho \leqq .154 \text { or } \rho \geqq .699 \text {. }
$$

While these bounds fall short of the conjectured result

$$
S(\rho)<\frac{1}{2} \ln 2 \text {, if } \rho<\frac{1}{4} \quad \text { or if } \rho>\frac{3}{8},
$$

they are considerably better than the earlier bounds. These can be improved somewhat by a more extensive numerical calculation. A substantial improvement will perhaps require a different technique. In this paper, we report these bounds.

More recently Kleitman and Rothschild [3] have been able to determine $S(\rho)$ exactly in the range $0 \leqq \rho \leqq 1 / 4$. Their results, in particular, imply the first part of the inequality (5). Their result is obviously better than our bound for $\rho<.154$. However, their method does not seem to be generalizable to higher $\rho$ values.

II. Preliminaries and notation. Consider a set $\mathscr{S}$, consisting of $n$ distinct elements. Let $R$ be a partial order relation defined on this set. We shall use the notation $a \geqq b(a, b \in \mathscr{S})$ iff $a$ is related to $b$ under $R$. We write $a>b$ iff $a \geqq b$ and $a \neq b$. A pair $(a, b)$ is said to be comparable iff $a \geqq b$ or $b \geqq a$. It is nontrivial if $a \neq b$.

An ordered sequence $a_{1}, a_{2}, a_{3}, \cdots, a_{l}$ of elements of $\mathscr{S}$ constitutes a chain of length $l$ iff $a_{1}>a_{2}>a_{3} \cdots>a_{l}$. The rank of an element $a$, denoted by $r(a)$, is defined as the length of the longest chain in $\mathscr{S}$ which starts with $a$. By $r(T)$ we shall denote the specification of rank of each of the elements of $T \subseteq \mathscr{S}$.

The 'relation $R$ induces a decomposition into maximal disjoint chains $C_{1}, C_{2}, \cdots, C_{p}$. (This decomposition need not be unique.) The chains $C_{i}$ are constructed as follows: $C_{1}$ is a longest chain in $\mathscr{S}$. $C_{2}$ is a longest chain in $\mathscr{S}-C_{1} . C_{3}$ is longest chain in $\mathscr{S}-C_{1}-C_{2}$, and so on. The process is continued till all the elements of $\mathscr{S}$ are exhausted. The length of a chain $C_{i}$ will be denoted by $l_{i}$. Clearly, we have $l_{1} \geqq l_{2} \geqq l_{3}, \cdots$. Also, any element of a chain $C_{i}$ is incomparable to at least one element in each of the preceding chains $C_{j}, j<i$.

Let $N_{n}(m)$ be the number of different partial order relations $R$ definable over $\mathscr{S}$, having exactly $m$ nontrivial, comparable pairs. Let $\Omega_{n}(z)$ be the generating function for $N_{n}(m)$, i.e.,

$$
\Omega_{n}(z)=\sum_{m=0}^{n(n-1) / 2} N_{n}(m) z^{m}
$$

Let $A$ and $B$ be disjoint, ordered subsets of $\mathscr{S}$. By an ordered subset here we mean a subset whose first, second elements 
are identified. Let $|A|=i$ and $|B|=j$. Also let $\widetilde{r}$ be a mapping from the set $A \cup B$ to the set of integers. Let $\widetilde{R}$ be a partial order relation on $A \cup B$. We say that $\widetilde{R}$ is consistent with the maximal chain structure $\{A, B\}$ and the rank function $\widetilde{r}$ iff there exists a partial order relation $R$ defined over $\mathscr{S}$ such that

(i) $\widetilde{R}$ is the restriction of $R$ to the domain $A \cup B$.

(ii) $A$ and $B$ are chains under some maximal chain decomposition of $\mathscr{S}$ under $R$.

(iii) For all $x \in A \cup B, \widetilde{r}(x)$ is the rank of $x$ under $R$. We now define

$$
\begin{aligned}
P_{k}(\widetilde{r} ; A, B)= & \text { The number of distinct partial order relations } \widetilde{R}, \\
& \text { which are consistent with the maximal chain struc- } \\
& \text { ture }\{A, B\} \text {, and the rank function } \widetilde{r} \text {, and have } \\
& \text { exactly } k \text { comparable pairs of the form }(a, b) \text { where } \\
& a \in A, b \in B .
\end{aligned}
$$

Clearly, $P_{k}(\widetilde{r} ; A, B)$ depends on the chains $A$ and $B$ only through their lengths. Hence, we may write

$$
P_{k}(\tilde{r} ; A, B)=P_{i j, k}(\widetilde{r}) .
$$

We further define the generating functions $P_{i j}(z)$ by

$$
P_{i j}(z)=P_{j i}(z)=\sum_{k=0}^{i j} z^{k} P_{i j, k},
$$

where

$$
P_{i j, k}=\max _{\tilde{r}}\left[P_{i j, k}(\widetilde{r})\right] .
$$

In eq. (9), the maximum is taken over all possible rank assignments. We shall assume that $n$ is sufficiently large so that $P_{i j, k}$ is independent of $n$.

These polynomials $P_{i j}(z)$ are easily determined for small values of $i$ and $j$, by exhaustive enumeration. Some low order polynomials are listed in the appendix, where an outline of the method used for their determination is also given. We used a computer program to determine all the polynomials for $i, j \leqq 6$. For higher values of $i$ and $j$, the computation time increases very sharply.

We define polynomial $\bar{P}_{i j}(z)$ similarly. These are generating functions $\bar{P}_{i j, k} \stackrel{\text { def }}{=} \operatorname{Max}_{\tilde{r}} \bar{P}_{i j, k}(\widetilde{r})$, where $\bar{P}_{i j, k}(\widetilde{r})$ is defined similar to $P_{i j, k}(\widetilde{r})$, except that we do not require the chains $A$ and $B$ to be maximal. These too were determined by exhaustive search.

The general properties of these polynomials $P_{i j}(z)$ and $\bar{P}_{i j}(z)$ are not very obvious. For large $i$, with $i / j$ held fixed, $P_{i j}(z) z^{-i j}$ is ex- 
pected to behave like $[g(z)]^{i}$, where $g(z)$ is some (as yet unknown) function of $z$. The study of these polynomials is interesting on its own, but not really necessary for our discussion here.

III. The upper bound. We may now state the main result of this paper.

THEOREM. Let $z, \rho, f_{i}$ by any nonnegative real numbers satisfying the following conditions:

(i) $\quad \sum_{i} i f_{i}=1$.

(ii) $0 \leqq \rho \leqq 1$.

Then $S(\rho) \leqq \operatorname{Max}_{\left\{f_{i}\right\}}\left[\sum_{i, j} f_{i} f_{j} \ln P_{i j}(z)-\rho \ln z\right]$.

Proof. Consider a particular decomposition of $\mathscr{S}$ in maximal disjoint chains $C_{1}, C_{2} \cdots C_{p}$. Let the lengths of these chains be $l_{1}, l_{2} \cdots l_{p}$ respectively, where $l_{1} \geqq l_{2} \geqq \cdots \geqq l_{p}$. Consider, also, a rank function $r(\mathscr{S})$.

Let $R^{\prime}$ be a binary relation defined over $\mathscr{S}$ satisfying the following property for all $i$ and $j$, the restriction of $R^{\prime}$ to the set $C_{i} U C_{j}$ is a partial order relation consistent with the maximal chain structure $\left\{C_{1}, C_{2} \cdots C_{p}\right\}$, and the rank function $r(\mathscr{S})$. Clearly, not all such relations $R^{\prime}$, define a partial order relation over the full set $\mathscr{S}$. The enumeration of all relations satisfying the above property, gives an upper bound on the enumeration of all partial order relations $R$ satisfying the above property. The relations $R^{\prime}$ are easily enumerated in terms of the polynomials $P_{i j}(z)$ defined earlier, and we get quite easily

$$
\Omega_{n}(z) \leqq \sum_{\left\{C_{i}\right\}} \sum_{r(\mathscr{G})}\left[z^{\Sigma_{\imath} l_{i}\left(l_{i}-1\right) / 2} \prod_{(i, j)}^{\prime} P_{l_{l} l_{j}}(z)\right]
$$

In this inequality, the summations over $\left\{C_{i}\right\}$ and over $r(\mathscr{S})$ are over all possible chain decompositions of $\mathscr{S}$ and all possible rank functions $r(\mathscr{S})$. The term $z^{l_{i}\left(l_{i}-1\right) / 2}$ comes from the $l_{i}\left(l_{i}-1\right) / 2$ comparable pairs in the chain $C_{i}$, and $P_{l_{i} l_{j}}(z)$ is the contribution of the mutual pairs between the chains $C_{i}$ and $C_{j}$. The prime over the product sign indicates the fact that $i=j$ term is excluded from the product. This inequality (10), clearly holds term by term for each power of $z$.

Now, the rank of an element in $\mathscr{S}$ can take values 1 to $n$. Hence total number of possible rank assignments is certainly less than $n^{n}$. Also, the total number of ways, the set $\mathscr{S}$ may be broken into disjoint subsets is at most ${ }^{2 n} P_{n}$. Hence we get from the inequality (10) 


$$
\Omega_{n}(z) \leqq{ }^{2 n} P_{n} n^{n} \operatorname{Max}_{\left\{l_{i}\right\}}\left[z^{\sum_{i} l_{i}\left(l_{i}-1\right) / 2} \prod_{(i, j)}^{\prime} P_{l_{i} l_{j}}(z)\right],
$$

where the maximal is taken over all possible partitions $\left\{l_{i}\right\}$ of $n$ $\left(\sum_{i} l_{i}=n\right)$.

$\Omega_{n}(z)$ is a polynomial in $z$ with positive coefficients. Hence for all real positive values of $z$

$$
N_{n}(m) \leqq \Omega_{n}(z) z^{-m} .
$$

Taking logarithms of both sides we get

$$
\begin{aligned}
& \ln N_{n}(m) \leqq \ln \left(n^{n}{ }^{2 n} P_{n}\right) \\
& +\operatorname{Max}_{\left\{l_{i}\right\}}\left[\sum_{i} \frac{l_{i}\left(l_{i}-1\right)}{2} \ln z-m \ln z+\sum_{\{i, j)}^{\prime} \ln P_{l_{i} l_{j}}(z)\right] .
\end{aligned}
$$

In the chain decomposition $\left\{C_{i}\right\}$, let the chains of length $i$ be $F_{i}$ in number. Since the total number of elements is $n$, we have

$$
\sum_{i} i F_{i}=n \text {. }
$$

The double summation on the right hand side of the inequality (13) may be rewritten as

$$
\sum_{(i, j)}^{\prime} \ln P_{l_{i} l_{j}}(z)=\sum_{i} \frac{F_{i}\left(F_{i}-1\right)}{2} \ln P_{i i}(z)+\sum_{i \neq j} \frac{F_{i} F_{j}}{2} \ln P_{i j}(z) .
$$

Substituting in (13) and taking the limit of large $n$, with $f_{i}=F_{i} / n$, we get

$$
S(\rho) \leqq \operatorname{Max}_{\left\{f_{i}\right\}}\left[\sum_{i, j} f_{i} f_{j} \ln P_{i j}(z)-\rho \ln z\right],
$$

which proves the theorem.

This theorem is not very useful for numerical calculation of upper bounds on $S(\rho)$, as knowledge of all the polynomials $P_{i j}(z)$ is required. For explicit calculation we use the following modified version of the theorem.

TheOREM. Let $p$ be any positive integer, and let $z, f_{i}(i=1$ to $p$ ), be any nonnegative real numbers satisfying the following conditions:

$$
\begin{aligned}
& \sum_{i=1}^{p} i f_{i}=1, \\
& 0 \leqq \rho \leqq 1,
\end{aligned}
$$




$$
S(\rho) \leqq \operatorname{Max}_{\left\lfloor f_{k}\right\}}\left[\sum_{i, j=1}^{p} f_{i} f_{j} \ln Q_{i j}(z)-\rho \ln z\right],
$$

where

$$
\begin{aligned}
& Q_{i j}(z)=P_{i j}(z), \quad \text { iff }(i \neq p \quad \text { and } \quad j \neq p), \\
& Q_{i j}(z)=\bar{P}_{i j}(z), \quad \text { iff }(i=p \quad \text { or } \quad j=p) .
\end{aligned}
$$

Proof. Express $\mathscr{S}$ as a union of disjoint chains of length less than or equal to $p$. Then the chains of length $p$ need not be maximal. Rest of the proof is as before.

We use variational calculus to maximize the right hand side of inequality (18), and then minimize the result with respect to $z$, to get the best upper bound. The constraint (17) is taken care of by a Lagrange multiplier. This gives the equations

$$
\rho=z \frac{\partial}{\partial z} \sum_{i, j=1}^{p} f_{i} f_{j} \ln Q_{i j}(z),
$$

and

$$
\sum_{j=1}^{p}\left[\ln Q_{i j}(z)\right] f_{j}=\lambda i, \quad \text { if } \quad f_{i}>0 .
$$

Here $\lambda$ is the Lagrange multiplier. Corresponding to any value of $z$, we first determine $f_{i}$ by solving the linear equations (17) and (22); and substitute in (21) and (18) to get the corresponding value $\rho$ and $S(\rho)$. By varying $z$, bounds for different values of $\rho$ are obtained. If for any value of $z$, the solution of equation (17) and (22) gives negative values of $f_{i}$ for some $i$, we choose that $f_{i}$ to be exactly zero and variationally optimize over the remaining variables. For $p=6$, the numerical results show that

$$
S(\rho)<\frac{1}{2} \ln 2, \text { if } \rho \leqq .154 \text { or if } \rho>.699,
$$

which is the promised result.

\section{APPENDIX}

Let $A=\left\{a_{1}, a_{2}, \cdots, a_{i}\right\}$ and $B=\left\{b_{1}, b_{2}, \cdots, b_{j}\right\}$. The rank function $\widetilde{r}$ on $A U B$ may be specified by a list of the form $a_{1} a_{9} b_{1} a_{3} b_{2} \cdots$, where the elements are arranged in order of decreasing rank. Consistency with the rank $r$ implies that no element can be greater than any element preceding it in the rank list. The exact values of ranks assigned are not relevant. The total number of rank functions to be tested is thus ${ }^{i+j} C_{j}$.

The relation $R$ may be represented by two lists, of the same 
form as the rank list. For $x, y \in A U B, x \geqq y$ if $x$ appears before $y$ in both of these lists. The computer program generates all possible relations $R$, and rejects those inconsistent with the rank list.

To save computation time, the maximality constraint was replaced by the following weaker constraint: If $i \geqq j$, then $a_{p} \ngtr b_{j-i+p}$ and $a_{p} \nless b_{p}$ for all $p$. If this condition fails, clearly the A chain is not maximal, as we can form a chain of length $(i+1)$ from $A U B$. Clearly, this relaxation of constraints does not affect the validity of the bounds derived. We list below some lower order polynomials $P_{i j}(z)$ and $\bar{P}_{i j}(z)$.

$P_{11}(z)=1$

$P_{12}(z)=1+2 z$

$P_{22}(z)=1+2 z+z^{2}$

$P_{13}(z)=1+2 z+2 z^{2}$

$P_{23}(z)=1+2 z+5 z^{2}+6 z^{3}+4 z^{4}$

$P_{33}(z)=1+2 z+5 z^{2}+6 z^{3}+6 z^{4}+4 z^{5}+z^{6}$

$\bar{P}_{11}(z)=1+z$

$\bar{P}_{12}(z)=1+2 z+z^{2}$

$\bar{P}_{16}(z)=1+2 z+3 z^{2}+4 z^{3}+3 z^{4}+2 z^{5}+z^{6}$

$\bar{P}_{26}(z)=1+2 z+5 z^{2}+8 z^{3}+14 z^{4}+18 z^{5}+22 z^{6}+22 z^{7}+21 z^{8}+16 z^{9}$

$+10 z^{10}+4 z^{11}+z^{12}$

\section{REFERENCES}

1. D. Dhar, Entropy and phase transitions in partially ordered sets, J. Math. Phys., 19 (1978), 1711-1713.

2. D. Kleitman and B. Rothschild, The number of finite topologies, Proc. Amer. Math. Soc., 25 (1970), 276-282.

3. - A phase transition on partial orders, Physica, 96A (1979), 254-259.

Received July 17, 1979.

Tata Institute of Fundamental Research

Bombay 400005, India 



\section{PACIFIC JOURNAL OF MATHEMATICS}

\section{EDITORS}

DONALD BABBITT (Managing Editor)

University of California

Los Angeles, CA 90024

Hugo RossI

University of Utah

Salt Lake City, UT 84112

C. C. MOORE and ANDREW OGG

University of California

Berkeley, CA 94720

\section{J. DugunduI}

Department of Mathematics

University of Southern California

Los Angeles, CA 90007

R. Finn and J. Milgram

Stanford University

Stanford, CA 94305

\section{ASSOCIATE EDITORS}
E. F. BeCKenBACH
B. H. NeumanN
F. WOLF
K. YosHIDA

\section{SUPPORTING INSTITUTIONS}

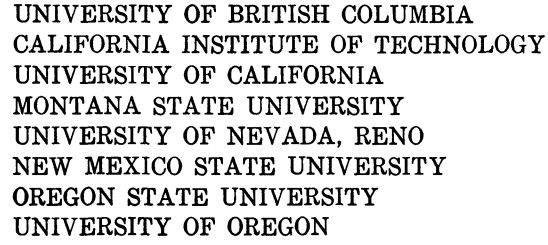

UNIVERSITY OF BRITISH COLUMBIA CALIFORNIA INSTITUTE OF TECHNOLOGY UNIVERSITY OF CALIFORNIA

MONTANA STATE UNIVERSITY

UNIVERSITY OF NEVADA, RENO

NEW MEXICO STATE UNIVERSITY

OREGON STATE UNIVERSITY UNIVERSITY OF OREGON

\author{
UNIVERSITY OF SOUTHERN CALIFORNIA \\ STANFORD UNIVERSITY \\ UNIVERSITY OF HAWAII \\ UNIVERSITY OF TOKYO \\ UNIVERSITY OF UTAH \\ WASHINGTON STATE UNIVERSITY \\ UNIVERSITY OF WASHINGTON
}

The Supporting Institutions listed above contribute to the cost of publication of this Journal, but they are not owners or publishers and have no responsibility for its content or policies.

Mathematical papers intended for publication in the Pacific Journal of Mathematics should be in typed form or offset-reproduced, (not dittoed), double spaced with large margins. Please do not use built up fractions in the text of the manuscript. However, you may use them in the displayed equations. Underline Greek letters in red, German in green, and script in blue. The first paragraph or two must be capable of being used separately as a synopsis of the entire paper. Please propose a heading for the odd numbered pages of less than 35 characters. Manuscripts, in triplicate, may be sent to any one of the editors. Please classify according to the scheme of Math. Reviews, Index to Vol. 39. Supply name and address of author to whom proofs should be sent. All other communications should be addressed to the managing editor, or Elaine Barth, University of California, Los Angeles, California, 90024.

50 reprints to each author are provided free for each article, only if page charges have been substantially paid. Additional copies may be obtained at cost in multiples of 50 .

The Pacific Journal of Mathematics is issued monthly as of January 1966. Regular subscription rate: $\$ 84.00$ a year (6 Vols., 12 issues). Special rato: $\$ 42.00$ a year to individual members of supporting institutions.

Subscriptions, orders for numbers issued in the last three calendar years, and changes of address shoud be sent to Pacific Journal of Mathematics, P.O. Box 969, Carmel Valley, CA 93924, U.S.A Old back numbers obtainable from Kraus Periodicals Co., Route 100, Millwood, NY 10546.

PUBLISHED BY PACIFIC JOURNAL OF MATHEMATICS, A NON-PROFIT CORPORATION

Printed at Kokusai Bunken Insatsusha (International Academic Printing Co., Ltd.). 8-8, 3-chome, Takadanobaba, Shinjuku-ku, Tokyo 160, Japan.

Copyright (C) 1980 by Pacific Jounal of Mathematics Manufactured and first issued in Japan 


\section{Pacific Journal of Mathematics \\ Vol. 90, No. $2 \quad$ October, 1980}

Frank Hayne Beatrous, Jr., Hölder estimates for the $\bar{\partial}$ equation with a support condition ..................................... 249

Charles L. Belna, Michael Jon Evans and Paul Humke, Planar continua

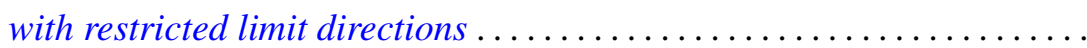

Leon Brown and Takashi Ito, Classes of Banach spaces with unique isometric preduals................................. 261

V. K. Deshpande, Completions of Noetherian hereditary prime rings ..... . 285

Deepak Dhar, Asymptotic enumeration of partially ordered sets . . . . . . . 299

Zeev Ditzian, On interpolation of $L_{p}[a, b]$ and weighted Sobolev

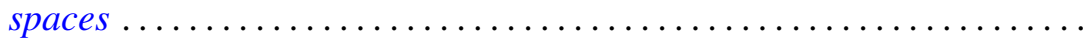

Andrew George Earnest, Congruence conditions on integers represented by

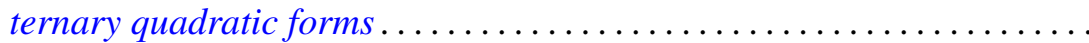

Melvin Faierman, Bounds for the eigenfunctions of a two-parameter system of ordinary differential equations of the second order ..............

Hector O. Fattorini, Vector-valued distributions having a smooth

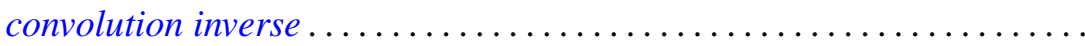

Howard D. Fegan, The spectrum of the Laplacian on forms over a Lie

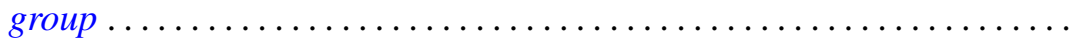

Gerald Leonard Gordon, On the degeneracy of a spectral sequence associated to normal crossings..

S. Madhavan, On bisimple weakly inverse semigroups ... 397

Françoise Mathot, On the decomposition of states of some

Roger McCann, Embedding asymptotically stable dynamical systems into radial flows in $l_{2}$

Michael L. Mihalik, Ends of fundamental groups in shape and proper homotopy...............................

Samuel Murray Rankin, III, Boundary value problems for partial functional differential equations .

Randy Tuler, Arithmetic sums that determine linear characters on $\Gamma(N)$

Jeffrey D. Vaaler, On linear forms and Diophantine approximation ..

G. P. Wene, Alternative rings whose symmetric elements are nilpotent or a right multiple is a symmetric idempotent. . 